\title{
Endothelial cell-initiated extravasation of cancer cells visualized in zebrafish
}

The extravasation of cancer cells, a key step for distant metastasis, is thought to be initiated by disruption of the endothelial barrier by malignant cancer cells. An endothelial covering-type extravasation of cancer cells in addition to conventional cancer cell invasion-type extravasation was dynamically visualized in a zebrafish hematogenous metastasis model. The inhibition of VEGF-signaling impaired the invasion-type extravasation via inhibition of cancer cell polarization and motility. Paradoxically, the antiangiogenic treatment showed the promotion, rather than the inhibition, of the endothelial covering-type extravasation of cancer cells, with structural changes in the endothelial walls. These findings may be a clue to the full understanding of the metastatic process as well as the metastatic acceleration by anti-angiogenic reagents observed in preclinical studies. 


\section{Endothelial cell-initiated extravasation of cancer cells visualized in zebrafish}

3 Masamitsu Kanada ${ }^{1, a}$, Jinyan Zhang $^{1}$, Libo Yan $^{1}$, Takashi Sakurai ${ }^{2}$ and Susumu

4 Terakawa ${ }^{1, b, *}$

5

$6{ }^{1}$ Medical Photonics Research Center, Hamamatsu University School of Medicine, 1-20-1

7 Handayama, Higashi-ku, Hamamatsu, 431-3192, Japan

$8{ }^{2}$ Electronics-inspired Interdiciplinary Research Institute, Toyohashi University of Technology, 1-1

9 Hibarigaoka, Tempaku, Toyohashi, 441-8580, Japan

10

$11{ }^{*}$ To whom correspondence may be addressed. E-mail: terakawa@sz.tokoha-u.ac.jp

$12{ }^{a}$ Present address: Department of Pediatrics, Stanford University School of Medicine, Clark

13 Center E150, 318 Campus Drive, Stanford, CA 94305, USA

14 b Present address: Department of Health Science, Tokoha University, 1-22-1 Sena, Aoi-ku,

15 Shizuoka, 420-0911, Japan

16

17 


\section{Abstract}

19 The extravasation of cancer cells, a key step for distant metastasis, is thought to be initiated by

20 disruption of the endothelial barrier by malignant cancer cells. An endothelial covering-type

21 extravasation of cancer cells in addition to conventional cancer cell invasion-type extravasation

22 was dynamically visualized in a zebrafish hematogenous metastasis model. The inhibition of

23 VEGF-signaling impaired the invasion-type extravasation via inhibition of cancer cell

24 polarization and motility. Paradoxically, the anti-angiogenic treatment showed the promotion,

25 rather than the inhibition, of the endothelial covering-type extravasation of cancer cells, with

26 structural changes in the endothelial walls. These findings may be a clue to the full understanding

27 of the metastatic process as well as the metastatic acceleration by anti-angiogenic reagents 28 observed in preclinical studies.

29

30 


\section{Introduction}

32 Metastasis is the primary factor associated with the death of cancer patients. There is no

33 therapeutic agent available to prevent this pathological step [1]. Metastatic progression proceeds

34 by multiple steps: first, the development of vasculature inside a primary nest of tumor,

35 intravasation of tumor cells into the newly developed leaky vasculature, survival of the cells

36 under the stress in the systemic circulation, extravasation of the cells from the circulation, and

37 finally proliferation at a secondary site in a distant tissue [2]. These steps have been verified by

38 studies of cancer cells or endothelial cells under in vitro culture conditions, or by examining

39 preparations of fixed tissue specimens. Although histological or biochemical techniques may

40 provide important information, such information is only validated at a certain point of time and

41 thus compromises the interpretation on the dynamic aspects of metastasis. One of the difficulties

42 in observing the behavior of cancer cells in vivo in mice by conventional high-resolution imaging

43 techniques is the low transparency of the tissue. Advanced techniques for intravital observations,

44 such as two-photon microscopies, imaging chamber recording, fiber-optic fluorescence

45 microendoscopies, have gradually enabled the visualization of the dynamic environmental

46 changes accompanying tumor development at a cellular level [3-5]. However, no study has so far

47 clearly shown the whole process of metastasis in mammalian tumor models at the cellular level.

48 A novel imaging technique was developed to overcome these difficulties in observing the

49 dynamic process of cancer cell metastasis in vivo, taking advantage of the high transparency of

50 zebrafish [6-8]. The zebrafish is an ideal vertebrate model for imaging, not only because of its

51 optical transparency but also because a comparison of the zebrafish genome with human's

52 revealed a remarkable conservation in the sequence of genes associated with the cell cycle, tumor

53 suppression, proto-oncogenes, angiogenic factors, and extracellular matrix proteins [9-11].

54 Highly metastatic cancer cells are often trapped in the capillaries and efficiently extravasated in 
55 the zebrafish, and an overexpression of the pro-metastatic gene "Twist" in cancer cells

56 dramatically promotes their intravascular migration and extravasation [7].

57 The present study extended the zebrafish hematogenous metastasis model, and thereby made

58 it possible to study the extravasation of human cancer cells especially after forming severe

59 emboli in the arterioles of zebrafish. The results obtained using a long-time fluorescence time-

60 lapse recording system demonstrate that human cancer cells extravasate according to the manner

61 generally accepted as an active invasion of a cancer cells. An extraordinary event was that a mass

62 of cancer cells underwent embolus formation and then also extravasated via a covering with a

63 layer of endothelial cells even in the absence of active invasion of the cancer cells. An electron

64 microscopic study of a mouse lung metastasis model revealed similar cancer cell extravasation

65 many years ago [12]. A dynamic observation method demonstrated that the covering by

66 endothelial cells is the major event in cancer cell extravasation. Furthermore, the live observation

67 system confirmed that VEGF was associated with this manner of extravasation. Paradoxically,

68 the treatment with an anti-angiogenic inhibitor shows the promotion, rather than the prevention,

69 of the endothelial covering-type extravasation.

70 


\section{PeerJ Reviewing Manuscript}

\section{Materials and Methods}

\section{Cell lines}

73 DsRed2 (referred to as RFP) expressing HeLa cells (obtained from Anticancer) were cultured in

74 RPMI-1640 supplemented with 10\% FBS, $2 \mathrm{mM}$ l-glutamine (Invitrogen), 1\% Penicillin-

75 Streptomycin (Invitrogen). The cells were incubated at $37^{\circ} \mathrm{C}$ in $5 \% \mathrm{CO}_{2}$ in a humidified 76 incubator.

\section{Zebrafish hematogenous metastasis model}

79 Maintenance of the transgenic zebrafish and the experimental design for this study were approved

80 by the Hamamatsu University School of Medicine animal welfare. Zebrafish were maintained

81 according to standard methods [13]. The transgenic strain of zebrafish expressing enhanced green

82 fluorescent protein (EGFP) under the $f l k 1$ (VEGFR2) promoter (flk1: EGFP) was obtained from

83 the Zebrafish International Resource Center [14]. Human cancer cells were microinjected into the

84 zebrafish larvae, following the protocol reported previously with some modifications [7]. Fish

85 larvae were dechorionated and anesthetized with $0.006 \%$ tricaine $48 \mathrm{~h}$ post-fertilization (hpf;

86 Sigma). Anesthetized larvae were then transferred onto an agarose gel for microinjection. RFP

87 expressing cancer cells were detached from culture dishes using Cell Dissociation Buffer

88 Enzyme-Free PBS-based (13151-014, Gibco) and then were washed twice with PBS. Cancer

89 cells were injected into the common cardinal vein using a tapered borosilicate glass capillary (1.0

$90 \mathrm{~mm}$ in diameter, World Precision Instruments, Inc.) with a tip diameter of $20-40 \mu \mathrm{m}$ (i.d.)

91 connected to a $50 \mathrm{ml}$ glass syringe. The position of the capillary tip was controlled by a

92 manipulator (M-152, Narishige Scientific). The injected fish larvae were kept at $32^{\circ} \mathrm{C}$ in the

93 presence of $25 \mu \mathrm{g} / \mathrm{ml}$ dexamethasone (Sigma) for $5-7 \mathrm{~h}$ for immunosuppression only before

94 observation because the presence of both tricaine and dexamethasone suppressed the heart beat of 


\section{PeerJ Reviewing Manuscript}

95 larvae. The larvae that formed severe emboli in the arterioles were selected under a fluorescence

96 stereomicroscope (SZX16, Olympus), and used for further experiments.

97

98 Live imaging of embolus-forming cancer cells and endothelial cells

99 A small drop of water containing an anesthetized larva was placed in a glass-bottom dish $(36 \mathrm{~mm}$,

100 Matsunami, Gifu). One $\mathrm{ml}$ of low temperature melting agarose (E-3126-25, BM equipment,

101 Tokyo) containing $0.006 \%$ tricaine was added to the dish to hold the larva in a gel attached to the

102 glass at the bottom. The dish was filled with $2 \mathrm{ml}$ water containing $0.006 \%$ tricaine. The long

103 time dual color time-lapse recording was carried out by using a homemade imaging system

104 driven by ImageJ software (NIH, USA) or a microscope system in an incubator (BioStation,

105 Nikon, Tokyo). The filter wheels (FW102, Tholabs, NJ) were controlled by the ImageJ software

106 (Research Services Branch, National Institute of Mental Health, Bethesda, Maryland, USA;

$107 \mathrm{http}: / /$ imagej.nih.gov/) through an IJSerial plugin (http://www.eslide.net/ijstage.php) for the dual

108 color fluorescence imaging, and time-lapse images were captured using a QuickTime Capture

109 plugin and Time-Lapse Video macros with some modifications

110 (http://rsbweb.nih.gov/ij/plugins/qt-capture.html). The analog monochrome camera (WAT-

$111120 \mathrm{~N}+$, Watec, Tokyo) was connected to the digital converter (ADVC-300, Canopus) and a PC

112 via IEEE1394 interface. The background noise was decreased using an image processor

113 (ARGUS-20, Hamamatsu Photonics, Hamamatsu). The temperature on the stage was maintained

114 at $32^{\circ} \mathrm{C}$ using a transparent heating plate (Kitazato, Fuji). In a standard recording, fluorescence

115 images were captured every $5 \mathrm{~min}$ for a total time of up to $11 \mathrm{~h}$. The spatial evaluation of cancer

116 cells and endothelial cells was carried out using a confocal microscope (FV1000, Olympus). 3D

117 stack images were taken by confocal microscopy (FV1000, Olympus) and processed using image

118 processing package Fiji (http://fiji.sc/Fiji) [15]. 
RNA interference, RT-qPCR

121 The siRNA sequence targeting human VEGF-A (referred here as VEGF) was the same as that

122 designed in a previous report [16]: 5'-GGAGUACCCUGAUGAGAUCdTdT-3' (sense), 5'-

123 GAUCUCAUCAGGGUACUCCdTdT-3' (antisense). MISSION siRNA Universal Negative

124 Control (Sigma) was used as a nonsense control siRNA. Relative mRNA amounts were

125 quantified using $\mathrm{iQ}^{\mathrm{TM}}$ SYBR Green supermix (Bio-Rad Laboratpries, Inc). Forward 5'-

126 CCTGGTGGACATCTTCCAGGAGTA-3' and reverse

127 CTTGGTGAGGTTTGATCCGCATAA-3' primers were used to detect VEGF, and forward 5'128 AACGGATTTGGTCGTATTGGGC-3' and reverse 5'-TTCTCAGCCTTGACGGTGCCAT-3' 129 primers were used to detect glyceraldehyde-3-phosphate dehydrogenase (GAPDH) mRNA. For

130 comparative, quantitative analysis, transcript levels were normalized to the level of GAPDH and 131 changes were determined. The comparative quantitation method $(\Delta \Delta \mathrm{Ct})$ was used to compare the 132 different samples and transformed to absolute values with $2^{\wedge}-\Delta \Delta C$ t for obtaining relative fold 133 changes. All assays were performed in triplicates. VEGF siRNA-treated RFP-HeLa cells were 134 injected into the blood vessels 17 - $19 \mathrm{~h}$ after transfection. The cancer cell-injected larvae were $135 \mathrm{kept}$ at $32^{\circ} \mathrm{C}$ in the presence of $25 \mu \mathrm{g} / \mathrm{ml}$ dexamethasone for $5-7 \mathrm{~h}$ for immunosuppression, and 136 these cells were then observed for $11 \mathrm{~h}$ within $37 \mathrm{~h}$ after the transfection, so that VEGF was 137 efficiently depleted during the observation period.

139 Anti-VEGF treatment

140 Sunitinib (Toronto Research Chemicals, Toronto), an orally active VEGFR tyrosine kinase

141 inhibitor, was dissolved in dimethyl sulfoxide (DMSO) to make a stock solution of $10 \mathrm{mM}$. The 142 stock solution was diluted in water to attain a concentration of $5 \mu \mathrm{M}$. Sunitinib treatment was 143 started immediately after cancer cell injection. The injected fish larvae were kept at $32^{\circ} \mathrm{C}$ in the 144 presence of $25 \mu \mathrm{g} / \mathrm{ml}$ dexamethasone (Sigma) and $5 \mu \mathrm{M}$ sunitinib for $5-7 \mathrm{~h}$ for

145 immunosuppression before observation. The cancer cell-injected larvae were then held in glass-

146 bottom dishes with low temperature melting agarose on which water containing $0.006 \%$ tricaine 147 and $5 \mu \mathrm{M}$ sunitinib was added. 
In vitro observation of live cells

150 The cells were seeded in polymer-bottom dishes (Bio Medical Science, Japan), and time-lapse

151 images were captured every minute for $12 \mathrm{~h}$ using BioStation (Nikon).

152

153 Immunofluorescence

154 The cells in the polymer-bottom dishes were fixed (4\% paraformaldehyde for $15 \mathrm{~min}$ ),

155 permeabilized (1\% Triton X-100 for $15 \mathrm{~min}$ ) and blocked (PBS containing 2\% bovine serum

156 albumin (Sigma, St Louis) for $30 \mathrm{~min}$ ) for non specific immunostaining. The cells were, then,

157 incubated with anti-vinculin mouse monoclonal antibody (ab18058, 1:200, Abcam, City)

158 overnight at $4^{\circ} \mathrm{C}$, subsequently with anti-mouse-IgG conjugated with Alexa 488 (Invitrogen), and

159 finally with $1 \mu \mathrm{g} / \mathrm{ml}$ Hoechst 33342 (Dojindo, Kamimashiki). The immunostained cells were

160 observed under a confocal microscope (FV1000, Olympus, Hachioji).

161

\section{Chemotactic cell migration assay}

163 The cells were seeded in BD Falcon ${ }^{\mathrm{TM}}$ FluoroBlok $^{\mathrm{TM}}$ 24-Multiwell Insert Systems (351157, BD,

164 NJ). The bottom wells were filled with chemoattractant-rich NIH3T3 conditioned medium. The

165 cells were stained with calcein-AM (Dojindo) after 24-h incubation, and the number of stained

166 cells in the bottom wells was counted in fluorescence images captured using an inverted

167 fluorescence microscope.

\section{Electron microscopy}

170 Fish larvae were fixed for scanning electron microscopy (SEM) by perfusion with $2 \%$

171 paraformaldehyde and 2.5\% glutaraldehyde in PBS using the microinjection system for

172 approximately $5 \mathrm{~min}$, and then transferred into the same fixation buffer. Samples were

173 dehydrated in a graded ethanol series, and frozen in liquid nitrogen and cracked into several 
174 pieces. These samples were then collected and freeze dried with t-butanol, and stained with $\mathrm{OsO}_{4}$,

175 and examined with a scanning electron microscope (S-4800, Hitachi, Tokyo).

176

177

178 Results

179 Extravasation of Embolus-forming Human Cancer Cells

180 This study first examined the potential for the extravasation of human cervical cancer cells

181 (HeLa). RFP-expressing HeLa (RFP-HeLa) cells were microinjected into the circulation,

182 following the protocol previously reported [7]. Unlike the previous study reporting that injected

183 cancer cells are arrested in the thinner intersegmental vessels [7], RFP-HeLa cells formed severe

184 emboli mostly in the thicker caudal artery immediately after injecting into the circulation. The

185 clusters of cancer cells in the emboli extravasated and adhered to the tissue outside the blood

186 vessels after 17 - $20 \mathrm{~h}$ (Figure 1A). The spatial distribution of the adhering-cancer cells and

187 blood vessel-forming endothelial cells was evaluated using a confocal microscope 1 day after the

188 cancer cell injection to confirm that embolus-forming cancer cells actually extravasated and

189 developed adhesion to the tissue outside the blood vessels. The optically sliced images clearly

190 showed that endothelial tube structures were devoid of the mass of cancer cells, and all of the

191 RFP-HeLa cells adhered to the tissue outside the blood vessels (Figure 1B). Therefore, RFP-

192 HeLa cells, which formed severe emboli in thicker caudal artery of zebrafish, have the ability to

193 efficiently extravasate and adhere to the tissues surrounding the blood vessels.

195 Two Processes of Extravasation in Human Cancer Cells

196 The process of extravasation by the RFP-HeLa cells was observed using a long-time dual color

197 time-lapse recording system to study mechanisms of extravasation of human cancer cells. The 
embolus-forming RFP-HeLa cells and endothelial cells could be observed for more than $11 \mathrm{~h}$,

199 which was long enough for these cells to exhibit slow behaviors. There were two distinctive

200 processes of extravasation observed in RFP-HeLa cells (Figure 2A). Some of the cancer cells

201 actively invaded into the vessel wall and penetrated the wall in the process broadly accepted as

202 the process of extravasation [7,17] (referred to as cancer cell invasion; Figure 2A, left; Movie

203 S1). In contrast, other masses of cancer cells seemed to be quiescent. These cells did not invade

204 the endothelial cell layer. Instead, a new leaf of endothelial cells appeared and extended over the

205 embolus-forming cells. The endothelial cells eventually covered the cancer cells on the vessel

206 wall. Simultaneously, the original layer of endothelial cells gradually disappeared and the cluster

207 of cancer cells spread to the tissue outside of the blood vessels (referred to as endothelial

208 covering; Figure 2A, right; Movie S2). Intriguingly, 9 out of 17 extravasations were carried out

209 in the absence of cancer cells' active invasion, while 5 were clearly accompanied by invasion of

210 cancer cells. In the remaining 3 extravasations, embolus-forming cancer cells showed invasion

211 and surrounding endothelial cells also newly extended over the cancer cells (Figure 2B). The

212 spatial distribution of embolus-forming cancer cells and newly spreading endothelial cells was

213 further analyzed using 3D confocal microscopic images (47 slices, step size: $1 \mu \mathrm{m}$ ) that were

214 taken at $10 \mathrm{~h}$ postadministration. Side views of the stack image and 3D reconstructed image

215 clearly showed that some endothelial cells were extending over the cancer cells or penetrating

216 into the cluster of cancer cells (Figure 2C; Movie S3). These results suggest that efficient

217 activation of endothelial cells by cancer cells is also important for extravasation, in addition to

218 high motility of cancer cells, which has been regarded as one of the most crucial factors

219 associated with malignancy.

220 Normal proliferating fibroblast $3 \mathrm{~T} 3$ cells (NIH, USA) showed neither the invasion type nor the

221 endothelial covering type extravasation in this model. Six clusters of these cells were in the 
222 vessels of 6 different individual fish for more than $11 \mathrm{~h}$. All of them formed severe emboli similar

223 to those of cancer cells, but showed neither the development of strong adhesions to the vascular

224 walls nor the process of extravasation mentioned above. These cells were eventually pushed away

225 by the blood flow. This suggests a difference to exist in the expression of adhesion molecules

226 between tumorigenic cells and normal cells, and adhesion to the endothelial walls is a key to

227 induce extravasation for the circulating cancer cells after forming an embolus in the blood 228 vessels.

\section{Effects of VEGF Depletion on Cancer Cell Properties and Extravasation}

231 Malignant tumors actively induce new blood vessels from surrounding tissues by secreting 232 vascular endothelial growth factor (VEGF) to receive nutrients and oxygen. In addition, cancer

233 cells may intravasate through this newly-formed leaky vasculature, leading to the metastasis in 234 remote places $[18,19]$. Hence we examined the effects of depletion of VEGF expressed in RFP-

235 HeLa cells to study the role of VEGF in tumor cell extravasation. The cells were transfected with 236 siRNA prior to their injection into the vessel of the fish. The efficient depletion of VEGF was 237 confirmed by real-time quantitative PCR (RT-qPCR). All the 3 family members of VEGFA, 238 which are secreted by cancer cells, have the same target sequence so that all the VEGFA (referred 239 to as VEGF, here) were depleted in the cancer cells. Expression of VEGF was depleted to $29 \pm$ $2406 \%$ in comparison to the control obtained by using nonsense siRNA-treated cells (Figure 3A). 241 The effect of VEGF depletion on the cancer cell itself was then evaluated morphologically in 242 vitro. The VEGF-depleted cells tended to aggregate with their motility decreased uniformly. They

243 were flattened and their adhering surface became very large (Figure 3B; Movie S5). Quantitative 244 evaluation indicated that cell motility was reduced to $36.0 \pm 7.7 \%$ in comparison to the control 245 obtained by using nonsense siRNA-treated cells as reported previously [20] (Figure 3C).

246 Vinculin, a focal adhesion protein, was immunostained in nonsense siRNA-treated and VEGF 
siRNA-treated RFP-HeLa cells to examine the effects of VEGF depletion on the adhesive

248 property of cancer cells. Focal adhesions were uniformly formed at the cell periphery in VEGF

249 depleted-cells, but only locally in control siRNA-treated cells so as to form a highly polarized

250 shape (Figure 3D). These results suggest that VEGF is involved not only in the activation of

251 endothelial cells, but also in the migration of cancer cells via cell polarization.

252 We next examined the effects of VEGF depletion on extravasation in vivo. Like the normal

253 RFP-HeLa cells, the VEGF-depleted cells immediately formed emboli after being injected into

254 blood vessels, and then the endothelial cells migrated over the embolus-forming cancer cells,

255 although the process was markedly delayed (Figure 3E). Strikingly, the process of cancer cell

256 invasion through the endothelial cells was completely suppressed during the 11-h observation

257 period (Figure 3E and 3F; Movie S6). Despite the severe inhibition of cancer cell invasion, six

258 extravasation events were still observed in VEGF-depleted cells (Figure 3F), thus suggesting that

259 the clinically adopted anti-angiogenic strategy of VEGF targeting is insufficient for the

260 prevention of metastasis. However, since RNA interference does not completely suppress target

261 molecule expression compared to that of gene knockout, we cannot rule out the possibility that

262 residual amount of VEGF could be enough to activate surrounding endothelial cells.

264 Effects of a Multi-targeted Anti-angiogenic Kinase Inhibitor on Cancer Cell

265 Properties and Extravasation

266 VEGF depletion in RFP-HeLa cells only delayed the endothelial covering-type extravasation,

267 while completely inhibiting the cancer cell invasion-type extravasation. Therefore we anticipated

268 that a potent inhibitor of tumor angiogenesis via VEGF signaling could completely inhibit both

269 types of extravasation. Sunitinib (Sutent ${ }^{\circledR}$, Pfizer Inc.), which effectively suppresses

270 angiogenesis by inhibiting signaling from the receptors for VEGF and for platelet derived growth

271 factor (PDGF) [21,22], was orally administered to the zebrafish larvae. The thinner 
272 intersegmental vessels were severely deteriorated by the overnight treatment with sunitinib

273 (Figure 4A). However, the thicker blood vessels were not affected morphologically, suggesting

274 that the effect of sunitinib is limited to the newly-formed vulnerable vessels. Endothelial cells in

275 the mature vasculature are independent of VEGF signaling for survival [23,24]. Sunitinib inhibits

276 the VEGF receptor, so the treatment of cells with sunitinib should affect the cancer cells like

277 VEGF depletion. As expected, the cells examined in vitro adhered to an obviously larger area of

278 the substrate, became less motile, and tended to aggregate in the presence of sunitinib, as the

279 VEGF-depleted cells did (Figure S1A). In addition, the sunitinib treatment reduced the cell

280 motility by $35.0 \pm 5.5 \%$ (Figure S1B). Focal adhesions were uniformly formed at the cell

281 periphery in sunitinib-treated cells, as in VEGF-depleted cells (Figure S1C).

282 The effect of sunitinib on extravasation was examined in vivo. Sunitinib treatment completely

283 suppressed the process of cancer cell invasion-type extravasation as expected. Unexpectedly, the

284 incidence of endothelial covering-type extravasation of RFP-HeLa cells was not affected by

285 treatment with sunitinib, although the process was markedly delayed, similar to the extravasation

286 of VEGF-depleted cells (Figure 4B; Movie S7). The area of cancer cells that were covered by

287 the endothelial cells in the presence of sunitinib was markedly larger than that in normal or

288 VEGF-depleted RFP-HeLa cells in most events of extravasation, and the blood vessel walls

289 concurrently, but slowly, moved toward the embolus-forming RFP-HeLa cells (Figure 4B;

290 Movie S7). Therefore, the sum of the area of extravasated cancer cells was calculated from 7

291 movies that were recorded in RFP-HeLa cells with and without sunitinib treatments to

292 quantitatively compare the whole volume of cancer cells extravasated, (Figure S1D). Although

293 the incidence of endothelial covering-type extravasation in the presence of sunitinib (9 incidences

294 in 7 larvae) was the same as that observed without treatment of sunitinib (Figure 2B and 4C),

295 sunitinib treatment increased the total volume of extravasated cancer cells to $153 \%$ of the volume

296 of extravasated cells without sunitinib treatment during 11-h observation period (Figure 4D). 
297 These results suggest that the anti-angiogenic inhibitor sunitinib can completely suppress the 298 cancer cell invasion-type extravasation without having strong effects on the incidence of 299 endothelial covering-type extravasation. Paradoxically, the total volume of the extravasated 300 cancer cells increased in the presence of sunitinib, thus suggesting that sunitinib accelerates the

301 process independently of the VEGF signaling. Moreover, the endothelial cells apparently 302 activated by sunitinib were studied morphologically using a scanning electron microscope. Many 303 protrusions were observed on the luminal face of endothelial walls of arteries in zebrafish, and 304 interestingly, many small holes were found in some regions on the endothelial wall (Figure 4F). 305 These holes are reminiscent of fenestrated endothelial walls in tumor vasculature or normal 306 microvasculature of mice [25-27]. Sunitinib-treated zebrafish larvae showed no hole on the 307 endothelial walls of arteries with fewer protrusions (Figure $\mathbf{4 H}$ ), and also showed prominently 308 thicker endothelial walls (Figure 4G). 


\section{Discussion}

311 Most reports addressing tumor metastasis present evidence supporting the assumption that

312 extravasation and intravasation are initiated by disruption of endothelial barrier by malignant

313 cancer cells via VEGF secretion and invasion [17]. The present study observed a new process,

314 potentially revising the conventional understanding of the mechanism of metastasis. A unique

315 hematogenous metastasis model in the transparent zebrafish was used to observe the

316 extravasation of human cancer cells after embolus formation at a cellular level. This approach

317 revealed that extravasation could be provoked by a mass of cancer cells, in addition to the

318 conventional manner that a single cell individually extravasates like the invasion of immune cells

319 [28]. The results confirmed that the extravasation is initiated by extension of new leaf of

320 endothelial cells covering the mass of tumor cells in the capillary [12]. The invasion of cancer

321 cells through the endothelium did occur as in the widely accepted model of cancer metastasis.

322 The study demonstrated that VEGF produced by the tumor cells is involved in regulation of their

323 migration by affecting the cell adhesion and polarization, and VEGF-depletion completely

324 suppressed the cancer cell invasion-type extravasation. On the other hand, the endothelial

325 covering-type extravasation was not inhibited but simply delayed by VEGF depletion, suggesting

326 that the process is partially dependent on VEGF and redundant pathways could compensate to

327 complete the extravasation. Sunitinib, an anti-angiogenic inhibitor, suppresses the migration and

328 proliferation of endothelial cells and the migration of the cancer cells simultaneously during the

329 extravasation. Surprisingly, sunitinib treatment had no significant effect on the process of

330 endothelial covering in the current metastasis model system, although it obviously inhibited the

331 new formation of intersegmental vasculature in the zebrafish. Furthermore, ultrastructural

332 observations of the luminal face of an artery in zebrafish showed that the sunitinib treatment

333 eliminated the fenestration-like holes on the endothelium and induced thicker vascular walls. 
334 These findings suggest that sunitinib induces vascular maturation independent of VEGF, while

335 deteriorates newly-formed vasculature. VEGF-independent vascular remodeling could be a key

336 regulatory mechanism underlying the extravasation of cancer cells. Since sunitinib is a multi

337 targeted kinase inhibitor, this vascular remodeling could be its effects on PDGFR or other targets.

338 To address this possibility, VEGF specific inhibition such as anti-VEGF antibody would be ideal,

339 although administrating adequate amount of antibody into the vasculature of small zebrafish

340 larvae is technically difficult.

341 The current findings also suggest that cancer cells have the potential to extravasate not only in

342 capillary vessels but also in mature blood vessels by forming clusters inducing vascular

343 remodeling. It is worth noting that extravasation of cancer cells as clusters is likely to increase the

344 chance of cancer cells to survive in the tissue outside the vasculature via secretion of trophic

345 factors for their growth or signal molecules for the immune tolerance in comparison to the

346 extravasation of a single cell. For these reasons, cancer cells' capability of activating endothelial

347 cells has to be taken into consideration as an aspect of malignancy, in addition to cancer cell

348 invasiveness.

349 Surprisingly, a VEGF-targeted inhibitor promoted the process of endothelial covering over

350 embolic cancer cells independently of VEGF, rather than inhibiting it. Similar evidence has been

351 presented in some preclinical studies, showing that VEGF-targeted inhibition promotes tumor

352 invasiveness and metastasis [29,30]. The explanation for these apparently paradoxical effects

353 were observed in these studies is still controversial [31]. Pàez-Ribes et al. (2009) demonstrated

354 that it appears to be an adaptive/evasive response by the tumor cells triggered by a disruption of

355 the tumor vasculature [30]. One plausible mechanism to trigger the adaptation of the tumor cells

356 is tumor hypoxia [32]. The anti-angiogenic treatment disrupts tumor vasculature in the initial

357 phase, but a mechanism of evasive resistance to the anti-angiogenic treatment is then switched on

358 to enable revascularization via alternative pro-angiogenic signals that increase local invasiveness, 
359 and/or enhance distant metastasis. Hypoxia is an effective driving force for the evasive resistance

360 of tumors through stabilization of hypoxia inducible factor-1 (HIF-1) [33]. Another study also

361 reported that sunitinib, a VEGF-targeted inhibitor, promotes tumor metastasis in a preclinical

362 model. Immunocompromised mice were pretreated or treated with sunitinib immediately after

363 intravenous inoculation with tumor cells [29]. Intriguingly, the anti-angiogenic treatment

364 increased the formation of metastatic foci, and shortened the overall survival time of the mice.

365 This acceleration of metastasis cannot be explained only by the mechanism of aforementioned

366 hypoxia-related effects on primary tumors. Therefore, the anti-angiogenic VEGF-targeted

367 inhibition may change the nature of vasculature increasing the probability of cancer cell lodging

368 and extravasation. The VEGF-disruption in mice can lead to a vessel disintegration, and render

369 the endothelium prothrombotic [24], which probably increases the number of places where cancer

370 cells lodge.

371 Many investigations reveal that there are multiple types of endothelial cells that have distinct

372 molecular signatures [27,34]. Notch pathway is implicated in the regulation of VEGF pathway,

373 and its up-regulation was suggested to contribute to VEGF-independent angiogenesis. This

374 reciprocal regulation of the endothelial cell types is explained using the tip/stalk model [35-37].

375 In addition, Mazzone et al. (2009) reported that a heterozygous deficiency of the oxygen-sensing

376 prolyl hydroxylase domain protein2 (PHD2) reverts the abnormal tumor vasculature formed in

377 the tumor-burdened mice to the mature and stable one, containing an orderly formed tight

378 monolayer of endothelial cell known as "phalanx cells" [27]. The sunitinib-treated endothelial

379 cells in our model are reminiscent of the "phalanx cells" in that the vascular walls, observed in

380 the sunitinib resistant monolayer endothelial cells in the current study. Based on the theory

381 regarding endothelial cell types and resistance to anti-angiogenic therapy, the current findings

382 provide a model to explain the VEGF-independent extravasation of cancer cells. The VEGF

383 dependency and fenestration reveals that 2 types of endothelial cell populations are present in the 
384 vasculature: one is the VEGF dependent and fenestrated-type, which are defined as active

385 endothelial cells that form new vasculature, and the other is the VEGF independent and

386 unfenestrated-type, which are quiescent endothelial cells that form a mature vasculature. The

387 application of anti-angiogenic inhibitors causes the new vasculature to deteriorate by apoptosis of

388 the active endothelial cells or by vascular maturation. In turn, the quiescent endothelial cells

389 could be reactivated for reconstruction of vessels independently of VEGF in order to maintain the

390 homeostasis of the vasculature (Figure 5). Some clinical or preclinical studies show that pro-

391 angiogenic factors other than VEGF are induced by the anti-angiogenic treatment [38-40]. This

392 evidence supports the current model that a disruption of neovascular endothelium by anti-

393 angiogenic drugs promotes endothelial covering-type extravasation via reactivation of quiescent

394 endothelial cells.

395 Anti-VEGF drugs such as the monoclonal anti-VEGF antibody bevacizumab [41,42] and the 396 multi-targeted receptor tyrosine kinase inhibitors sunitinib [43,44] and sorafenib [45,47] prolong

397 the life of some cancer patients, but the clinical benefits of the treatment are relatively modest

398 and usually prolong the overall survival of cancer patients by only months, without offering an

399 enduring cure [47,48], and in some cases it may shorten the survival by facilitating the tumor

400 invasiveness and metastasis. Although the mechanisms of the resistance to anti-angiogenic

401 treatments and acceleration of metastasis are still under investigation, intrinsic tumor resistance

402 or acquired resistance are proposed as possible mechanisms.

403 It is important to understand the complicated mechanism of vascular homeostasis during the

404 anti-angiogenic treatment of such tumors, because the effects of anti-angiogenic drugs on the

405 response of endothelial cells and on the intravasation and extravasation play a key role in each 406 step of metastasis.

407

408 
409

410

411

412

413

414

415

416

417

418

419

420

421

422

423

424

425

426

427

428

429

430

431

432

\section{Acknowledgements}

We would like to thank Ms. Yoko Kumakiri for her excellent technical assistance for the electron microscopic study, and Dr. Yuichi Hiratsuka for useful suggestion on ImageJ, and Dr. Kaoru Kato for useful suggestion on microinjection, and The Zebrafish International Resource Center for providing the transgenic zebrafish.

Figure 1. Extravasation of Cancer Cells after Severe Emboli Formation in Arterioles of Zebrafish Larvae. A, RFP-HeLa cells and endothelial cells in arterioles were examined under an epifluorescence microscope 17 - $20 \mathrm{~h}$ after formation of the emboli. B, The spatial locations of the extravasated RFP-HeLa cells and blood vessels were examined under a confocal microscope. Bars, $100 \mu \mathrm{m}(\mathrm{A})$ or $40 \mu \mathrm{m}(\mathrm{B})$.

Figure 2. Two Representative processes of Extravasation in Cancer Cells. A, Cancer cell invasion-type and endothelial covering-type extravasation in RFP-HeLa cells. Right panels show schematic outlines of the embolus-forming RFP-HeLa cells (red) and the surrounding endothelial cells (green). The numbers indicate the elapsed time in minutes. Bars, $40 \mu \mathrm{m}$. B, The incidence of the 2 processes of extravasation. Seven larvae in which RFP-HeLa cells formed severe emboli were observed. Seventeen extravasation events were counted during the $11 \mathrm{~h}$ observations. C, Side view of 3D reconstructed images. Some endothelial cells were extending over or penetrating through the embolus-forming RFP-HeLa cells (white arrows). Dashed lines in 2D image indicate locations of slicing along a vertical axis.

Figure 3. Effects of VEGF Depletion on Cancer Cell Properties and Extravasation. A, The 
433 depletion of VEGF in RFP-HeLa cells was confirmed by RT-qPCR $24 \mathrm{~h}$ after the transfection

434 with siRNA. GAPDH was amplified as an internal control. B, Phase contrast images of control

435 siRNA-treated and VEGF-depleted RFP-HeLa cells in culture. C, Quantitative evaluation of

436 chemotactic migration in control siRNA-treated and VEGF-depleted RFP-HeLa cells (mean \pm

$437 \mathrm{SD}, \mathrm{n}=3$ ). D, Confocal microscopic images of control siRNA-treated and VEGF-depleted RFP-

438 HeLa cells showing the distribution of vinculin (green) and DNA (blue). E, The extravasation of

439 VEGF-depleted RFP-HeLa cells. The numbers indicate the elapsed time in minutes. F, Incidence

440 of the 2 processes of extravasation. Nine larvae in which VEGF-depleted RFP-HeLa cells

441 formed severe emboli were observed. Six extravasation events were counted during $11 \mathrm{~h}$

442 observations. Bars, $20 \mu \mathrm{m}(\mathrm{B}, \mathrm{D})$ or $40 \mu \mathrm{m}(\mathrm{E})$.

444 Figure 4. Effects of Anti-angiogenic Inhibitor, Sunitinib, on the Vasculature of Zebrafish

445 Larvae and Cancer Cell Extravasation. A, Intersegmental vessels were severely deteriorated

446 by treatment with sunitinib. B, Extravasation of RFP-HeLa cells in the presence of sunitinib. The

447 numbers indicate the elapsed time in minutes. C, The incidence of the 2 processes of

448 extravasation. Seven larvae in which RFP-HeLa cells formed severe emboli were observed in the

449 presence of sunitinib. Nine extravasation events were counted during $11 \mathrm{~h}$ observations. D, The

450 sum of the area of extravasated cancer cell images was calculated from all of the 7 movies

451 recorded in RFP-HeLa cells with and without sunitinib treatments. Bars, $100 \mu \mathrm{m}$ (A) or $40 \mu \mathrm{m}$

452 (B). $\mathbf{E}$ and $\mathbf{G}$, Scanning electron micrographs of the arteries in the control and sunitinib-treated

453 larvae. Sunitinib-treated larvae showed thicker vascular walls. F, The magnified image of the

454 luminal face on the endothelial wall of a control larva. The endothelial wall showed many holes

455 or fenestration-like structures (arrowhead). H, The magnified image of the luminal face on the

456 endothelial wall of a sunitinib-treated larva. The endothelial wall showed no fenestration-like

457 structures. Bars, $10 \mu \mathrm{m}(\mathrm{E}$ and $\mathrm{G})$ or $0.5 \mu \mathrm{m}(\mathrm{F}$ and $\mathrm{H})$. 
Figure 5. Model of the endothelial coverage regulation via vascular homeostasis

460

461

462

463

464

465

466

467

468

469

470

471

472

473

474

475

476

477

478

479

480

481

482

483

484

485

486

487

488

489

490

491

492

493

494

495

496

497

maintenance. Active EC in the normal vasculature, which is VEGF-dependent and fenestrated, and quiescent EC, which is VEGF-independent and unfenestrated, coexist in the same blood vessel. VEGF-targeted inhibitors only affect the active ECs, thus resulting in deterioration of new vasculature via apoptosis or vascular maturation. In contrast, the quiescent ECs that contain mature vasculature are reactivated independently of VEGF in order to maintain vascular homeostasis.

\section{References}

1. Gupta GP, Massague J (2006) Cancer metastasis: Building a framework. Cell 127: 679-695.

2. Nguyen DX, Bos PD, Massague J (2009) Metastasis: from dissemination to organ-specific colonization. Nature Reviews Cancer 9: 274-U265.

3. Flusberg BA, Cocker ED, Piyawattanametha W, Jung JC, Cheung ELM, Schnitzer MJ (2005) Fiber-optic fluorescence imaging. Nature Methods 2: 941-950.

4. Beerling E, Ritsma L, Vrisekoop N, Derksen PWB, van Rheenen J (2011) Intravital microscopy: new insights into metastasis of tumors. Journal of Cell Science 124: 299-310.

5. Ritsma L, Steller EJ, Beerling E, Loomans CJ, Zomer A, Gerlach C, Vrisekoop N, Seinstra D, van Gurp L, Schafer R, Raats DA, de Graaff A, Schumacher TN, de Koning EJ, Rinkes IH, Kranenburg O, van Rheenen J (2012) Intravital microscopy through an abdominal imaging window reveals a pre-micrometastasis stage during liver metastasis. Science Translational Medicine 4: 158ra145.

6. Stoletov K, Montel V, Lester RD, Gonias SL, Klemke R (2007) High-resolution imaging of the dynamic tumor cell-vascular interface in transparent zebrafish. Proceedings of the National Academy of Sciences of the United States of America 104: 17406-17411.

7. Stoletov K, Kato H, Zardouzian E, Kelber J, Yang J, Shattil S, Klemke R (2010) Visualizing extravasation dynamics of metastatic tumor cells. Journal of Cell Science 123: 2332-2341.

8. Zhang J, Wei J, Kanada M, Yan L, Zhang Z, Watanabe H, Terakawa S (2013) Inhibition of store-operated $\mathrm{Ca}(2+)$ entry suppresses EGF-induced migration and eliminates extravasation from vasculature in nasopharyngeal carcinoma cell. Cancer Letter 336: 390397.

9. Berghmans S, Jette C, Langenau D, Hsu K, Stewart R, Look T, Kanki JP (2005) Making waves in cancer research: new models in the zebrafish. Biotechniques 39: 227-237.

10. Zon LI, Peterson RT (2005) In vivo drug discovery in the zebrafish. Nature Reviews Drug Discovery 4: 35-44.

11. Stoletov K, Klemke R (2008) Catch of the day: zebrafish as a human cancer model. Oncogene 27: 4509-4520.

12. Lapis K, Paku S, Liotta LA (1988) Endothelialization of embolized tumor cells during metastasis formation. Clinical \& experimental metastasis 6: 73-89.

13. Westerfield M (1993) The Zebrafish Book. Univ. Oregon Press, Eugene, Oregon. 
498

499

500

501

502

503

504

505

506

507

508

509

510

511

512

513

514

515

516

517

518

519

520

521

522

523

524

525

526

527

528

529

530

531

532

533

534

535

536

537

538

539

540

541

542

543

544

545

546

14. Jin S-W, Beis D, Mitchell T, Chen J-N, Stainier DYR (2005) Cellular and molecular analyses of vascular tube and lumen formation in zebrafish. Development (Cambridge, England) 132: 5199-5209.

15. Schindelin J, Arganda-Carreras I, Frise E, Kaynig V, Longair M, Pietzsch T, Preibisch S, Rueden C, Saalfeld S, Schmid B, Tinevez JY, White DJ, Hartenstein V, Eliceiri K, Tomancak P, Cardona A (2012) Fiji: an open-source platform for biological-image analysis, Nature Methods 9: 676-682.

16. Takei Y, Kadomatsu K, Yuzawa Y, Matsuo S, Muramatsu T (2004) A small interfering RNA targeting vascular endothelial growth factor as cancer therapeutics. Cancer Research 64: 3365-3370.

17. Weis S, Cui JH, Barnes L, Cheresh D (2004) Endothelial barrier disruption by VEGFmediated Src activity potentiates tumor cell extravasation and metastasis. Journal of Cell Biology 167: 223-229.

18. Senger DR, Vandewater L, Brown LF, Nagy JA, Yeo KT, Yeo TK, Berse B, Jackman RW, Dvorak AM, Dvorak HF (1993) VASCULAR-PERMEABILITY FACTOR (VPF, VEGF) IN TUMOR BIOLOGY. Cancer and Metastasis Reviews 12: 303-324.

19. Esser S, Lampugnani MG, Corada M, Dejana E, Risau W (1998) Vascular endothelial growth factor induces VE-cadherin tyrosine phosphorylation in endothelial cells. Journal of Cell Science 111: 1853-1865.

20. Bachelder RE, Lipscomb EA, Lin XN, Wendt MA, Chadborn NH, Eickholt BJ, Mercurio AM (2003) Competing autocrine pathways involving alternative neuropilin-1 Lligands regulate chemotaxis of carcinoma cells. Cancer Research 63: 5230-5233.

21. Bergers G, Song S, Meyer-Morse N, Bergsland E, Hanahan D (2003) Benefits of targeting both pericytes and endothelial cells in the tumor vasculature with kinase inhibitors. Journal of Clinical Investigation 111: 1287-1295.

22. Pietras K, Hanahan D (2005) A multitargeted, metronomic, and maximum-tolerated dose "chemo-switch" regimen is antiangiogenic, producing objective responses and survival benefit in a mouse model of cancer. Journal of Clinical Oncology 23: 939-952.

23. Gerber HP, Hillan KJ, Ryan AM, Kowalski J, Keller GA, Rangell L, Wright BD, Radtke F, Aguet M, Ferrara N (1999) VEGF is required for growth and survival in neonatal mice. Development 126: 1149-1159.

24. Lee S, Chen TT, Barber CL, Jordan MC, Murdock J, Desai S, Ferrara N, Nagy A, Roos KP, Iruela-Arispe ML (2007) Autocrine VEGF signaling is required for vascular homeostasis. Cell 130: 691-703.

25. Inai T, Mancuso M, Hashizume H, Baffert F, Haskell A, Baluk P, Hu-Lowe DD, Shalinsky DR, Thurston G, Yancopoulos GD, McDonald DM (2004) Inhibition of vascular endothelial growth factor (VEGF) signaling in cancer causes loss of endothelial fenestrations, regression of tumor vessels, and appearance of basement membrane ghosts. American Journal of Pathology 165: 35-52.

26. Kamba T, Tam BYY, Hashizume H, Haskell A, Sennino B, Mancuso MR, Norberg SM, O’Brien SM, Davis RB, Gowen LC, Anderson KD, Thurston G, Joho S, Springer ML, Kuo CJ, McDonald DM (2006) VEGF-dependent plasticity of fenestrated capillaries in the normal adult microvasculature. American Journal of Physiology-Heart and Circulatory Physiology 290: H560-H576.

27. Mazzone M, Dettori D, de Oliveira RL, Loges S, Schmidt T, Jonckx B, Tian YM, Lanahan AA, Pollard P, Ruiz de Almodovar C, De Smet F, Vinckier S, Aragones J, Debackere K, Luttun A, Wyns S, Jordan B, Pisacane A, Gallez B, Lampugnani MG, Dejana E, Simons M, Ratcliffe P, Maxwell P, Carmeliet P (2009) Heterozygous Deficiency of PHD2 Restores Tumor Oxygenation and Inhibits Metastasis via Endothelial Normalization. Cell

PeerJ reviewing PDF | (v2014:09:2694:1:1:NEW 11 Nov 2014) 
136: 839-851. extravasation: chemokine transport and presentation by the endothelium. Blood 100: 3853-3860.

29. Ebos JML, Lee CR, Cruz-Munoz W, Bjarnason GA, Christensen JG, Kerbel RS (2009) Accelerated Metastasis after Short-Term Treatment with a Potent Inhibitor of Tumor Angiogenesis. Cancer Cell 15: 232-239.

30. Paez-Ribes M, Allen E, Hudock J, Takeda T, Okuyama H, Vinals F, Inoue M, Bergers G, Hanahan D, Casanovas O (2009) Antiangiogenic Therapy Elicits Malignant Progression of Tumors to Increased Local Invasion and Distant Metastasis. Cancer Cell 15: 220-231.

31. Loges S, Mazzone M, Hohensinner P, Carmeliet P (2009) Silencing or Fueling Metastasis with VEGF Inhibitors: Antiangiogenesis Revisited. Cancer Cell 15: 167-170.

32. Brahimi-Horn MC, Chiche J, Pouyssegur J (2007) Hypoxia and cancer. Journal of Molecular Medicine-Jmm 85: 1301-1307.

33. Semenza GL (2003) Targeting HIF-1 for cancer therapy. Nature Reviews Cancer 3: 721-732.

34. Phng LK, Gerhardt H (2009) Angiogenesis: A Team Effort Coordinated by Notch. Developmental Cell 16: 196-208.

35. Noguera-Troise I, Daly C, Papadopoulos NJ, Coetzee S, Boland P, Gale NW, Lin HC, Yancopoulos GD, Thurston G (2006) Blockade of Dll4 inhibits tumour growth by promoting non-productive angiogenesis. Nature 444: 1032-1037.

36. Ridgway J, Zhang G, Wu Y, Stawicki S, Liang WC, Chanthery Y, Kowalski J, Watts RJ, Callahan C, Kasman I, Singh M, Chien M, Tan C, Hongo JA, de Sauvage F, Plowman G, Yan M (2006) Inhibition of D114 signalling inhibits tumour growth by deregulating angiogenesis. Nature 444: 1083-1087.

37. Scehnet JS, Jiang W, Kumar SR, Krasnoperov V, Trindade A, Benedito R, Djokovic D, Borges C, Ley EJ, Duarte A, Gill PS (2007) Inhibition of Dll4-mediated signaling induces proliferation of immature vessels and results in poor tissue perfusion. Blood 109: 47534760.

38. Willett CG, Boucher Y, Duda DG, di Tomaso E, Munn LL, Tong RT, Kozin SV, Petit L, Jain RK, Chung DC, Sahani DV, Kalva SP, Cohen KS, Scadden DT, Fischman AJ, Clark JW, Ryan DP, Zhu AX, Blaszkowsky LS, Shellito PC, Mino-Kenudson M, Lauwers GY (2005) Surrogate markers for antiangiogenic therapy and dose-limiting toxicities for bevacizumab with radiation and chemotherapy: Continued experience of a phase I trial in rectal cancer patients. Journal of Clinical Oncology 23: 8136-8139.

39. Ebos JML, Lee CR, Christensen JG, Mutsaers AJ, Kerbel RS (2007) Multiple circulating proangiogenic factors induced by sunitinib malate are tumor-independent and correlate with antitumor efficacy. Proceedings of the National Academy of Sciences of the United States of America 104: 17069-17074.

40. Casanovas O, Hicklin DJ, Bergers G, Hanahan D (2005) Drug resistance by evasion of antiangiogenic targeting of VEGF signaling in late-stage pancreatic islet tumors. Cancer Cell 8: 299-309.

41. Hurwitz H, Fehrenbacher L, Novotny W, Cartwright T, Hainsworth J, Heim W, Berlin J, Baron A, Griffing S, Holmgren E, Ferrara N, Fyfe G, Rogers B, Ross R, Kabbinavar F (2004) Bevacizumab plus irinotecan, fluorouracil, and leucovorin for metastatic colorectal cancer. New England Journal of Medicine 350: 2335-2342.

42. Miller K, Wang ML, Gralow J, Dickler M, Cobleigh M, Perez EA, Shenkier T, Cella D, Davidson NE (2007) Paclitaxel plus bevacizumab versus paclitaxel alone for metastatic breast cancer. New England Journal of Medicine 357: 2666-2676. 
615

616
G, Judson IR, Heinrich MC, Morgan JA, Desai J, Fletcher CD, George S, Bello CL, Huang X, Baum CM, Casali PG (2006) Efficacy and safety of sunitinib in patients with advanced gastrointestinal stromal tumour after failure of imatinib: a randomised controlled trial. Lancet 368: 1329-1338.

44. Motzer RJ, Michaelson MD, Redman BG, Hudes GR, Wilding G, Figlin RA, Ginsberg MS, Kim ST, Baum CM, DePrimo SE, Li JZ, Bello CL, Theuer CP, George DJ, Rini BI (2006) Activity of SU11248, a multitargeted inhibitor of vascular endothelial growth factor receptor and platelet-derived growth factor receptor, in patients with metastatic renal cell carcinoma. Journal of Clinical Oncology 24: 16-24.

45. Abou-Alfa GK, Schwartz L, Ricci S, Amadori D, Santoro A, Figer A, De Greve J, Douillard JY, Lathia C, Schwartz B, Taylor I, Moscovici M, Saltz LB (2006) Phase II study of sorafenib in patients with advanced hepatocellular carcinoma. Journal of Clinical Oncology 24: 4293-4300.

46. Escudier B (2007) Sorafenib in advanced clear-cell renal-cell carcinoma (vol 356, pg 125, 2007). New England Journal of Medicine 357: 203-203.

47. Grepin R, Pages G (2010) Molecular mechanisms of resistance to tumour anti-angiogenic strategies. Journal of oncology 2010: 835680.

48. Kerbel RS (2008) Molecular origins of cancer: Tumor angiogenesis. New England Journal of Medicine 358: 2039-2049. 


\section{Figure 1}

Extravasation of Cancer Cells after Severe Emboli Formation in Arterioles of Zebrafish Larvae.

A, RFP-HeLa cells and endothelial cells in arterioles were examined under an epifluorescence microscope 17 - $20 \mathrm{~h}$ after formation of the emboli. B, The spatial locations of the extravasated RFP-HeLa cells and blood vessels were examined under a confocal microscope. Bars, $100 \mu \mathrm{m}(\mathrm{A})$ or $40 \mu \mathrm{m}(\mathrm{B})$.
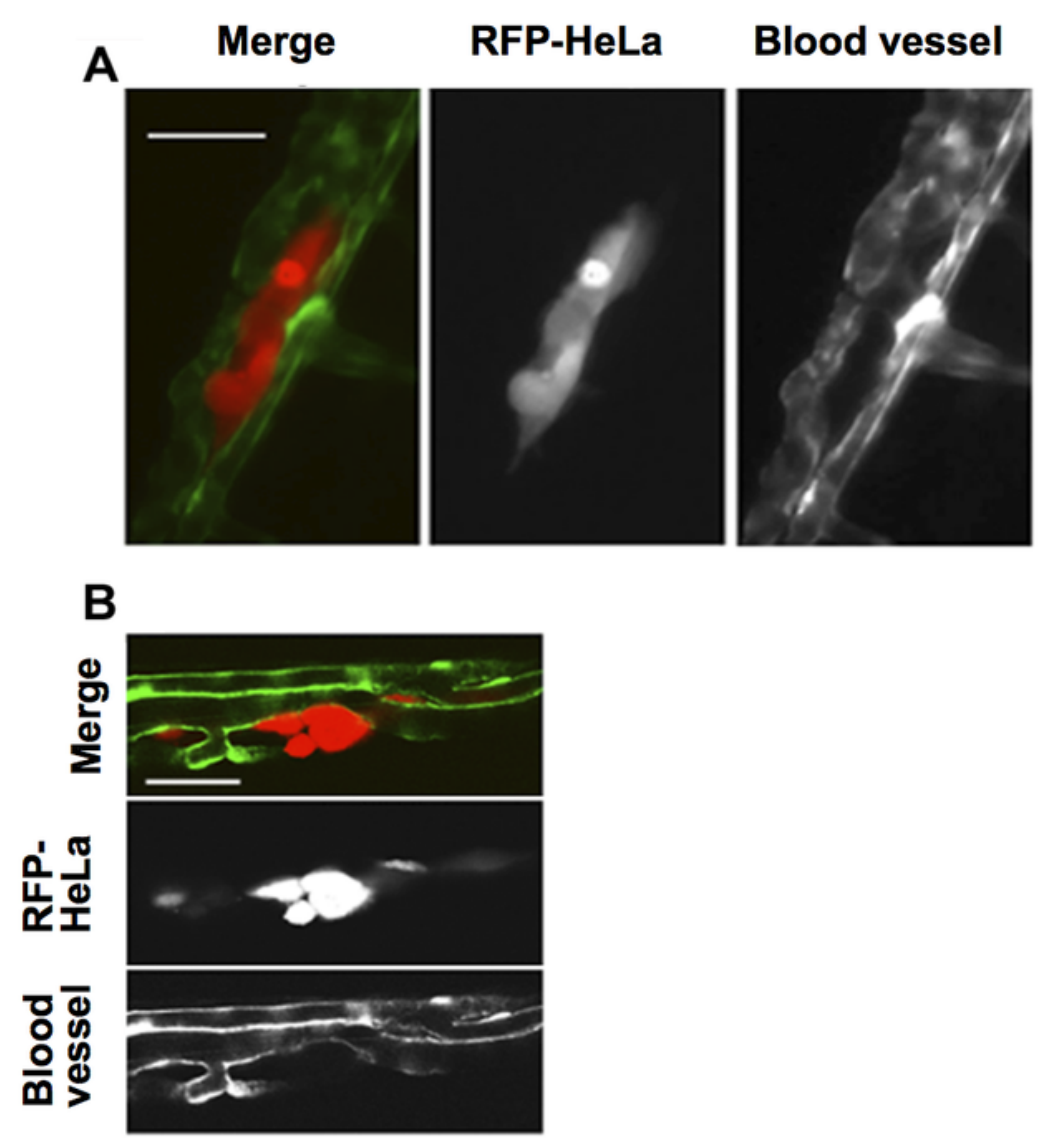


\section{Figure 2}

Two Representative processes of Extravasation in Cancer Cells.

A, Cancer cell invasion-type and endothelial covering-type extravasation in RFP-HeLa cells. Right panels show schematic outlines of the embolus-forming RFP-HeLa cells (red) and the surrounding endothelial cells (green). The numbers indicate the elapsed time in minutes. Bars, $40 \mu \mathrm{m}$. B, The incidence of the 2 processes of extravasation. Seven larvae in which RFP-HeLa cells formed severe emboli were observed. Seventeen extravasation events were counted during the $11 \mathrm{~h}$ observations. C, Side view of 3D reconstructed images. Some endothelial cells were extending over or penetrating through the embolus-forming RFP-HeLa cells (white arrows). Dashed lines in 2D image indicate locations of slicing along a vertical axis. 

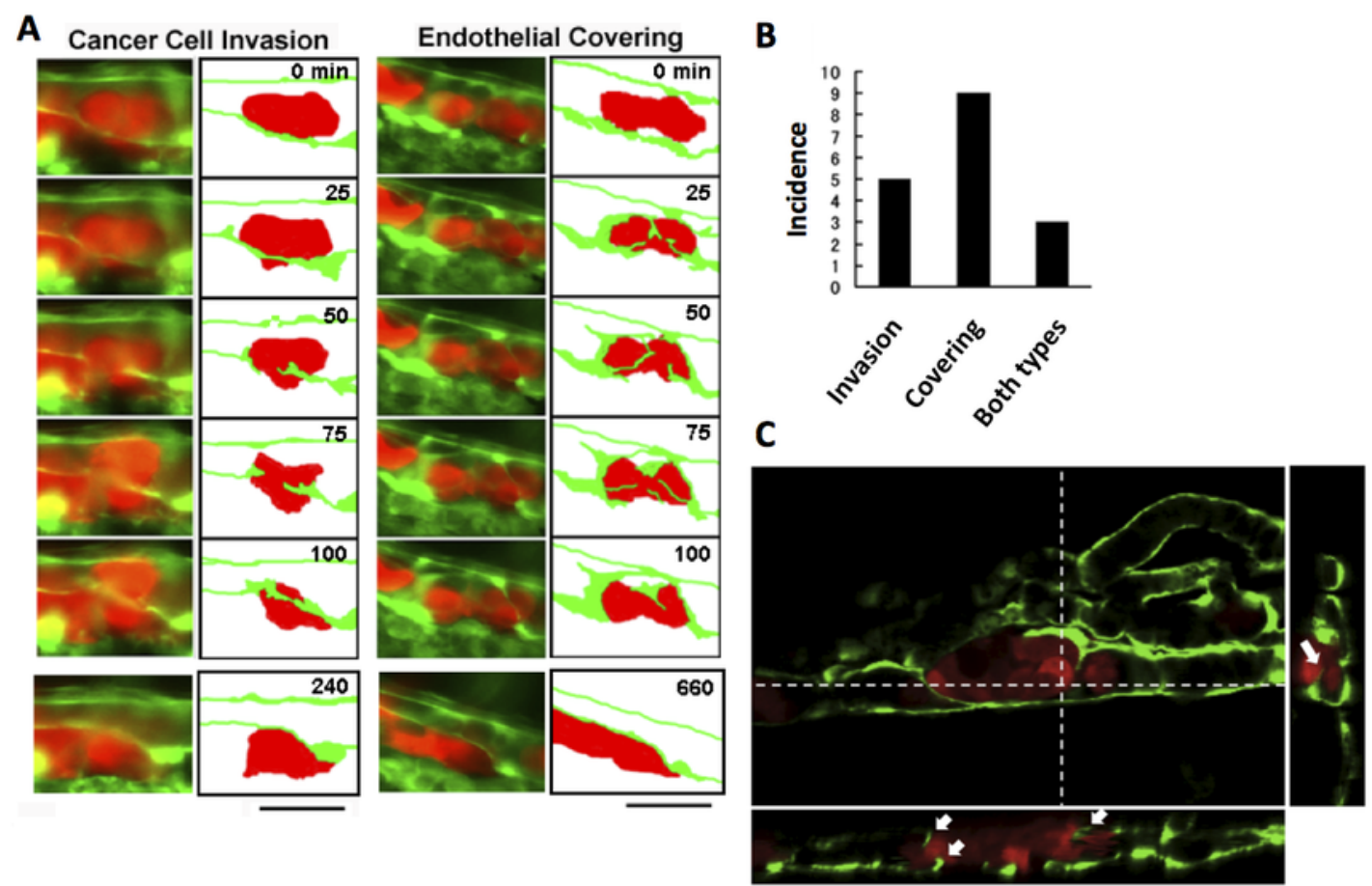


\section{Figure 3}

Effects of VEGF Depletion on Cancer Cell Properties and Extravasation.

A, The depletion of VEGF in RFP-HeLa cells was confirmed by RT-qPCR $24 \mathrm{~h}$ after the transfection with siRNA. GAPDH was amplified as an internal control. B, Phase contrast images of control siRNA-treated and VEGF-depleted RFP-HeLa cells in culture. C, Quantitative evaluation of chemotactic migration in control siRNA-treated and VEGF-depleted RFP-HeLa cells (mean $\pm S D, n=3$ ). D, Confocal microscopic images of control siRNA-treated and VEGFdepleted RFP-HeLa cells showing the distribution of vinculin (green) and DNA (blue). E, The extravasation of VEGF-depleted RFP-HeLa cells. The numbers indicate the elapsed time in minutes. $\mathbf{F}$, Incidence of the 2 processes of extravasation. Nine larvae in which VEGFdepleted RFP-HeLa cells formed severe emboli were observed. Six extravasation events were counted during $11 \mathrm{~h}$ observations. Bars, $20 \mu \mathrm{m}$ (B, D) or $40 \mu \mathrm{m}$ (E). 


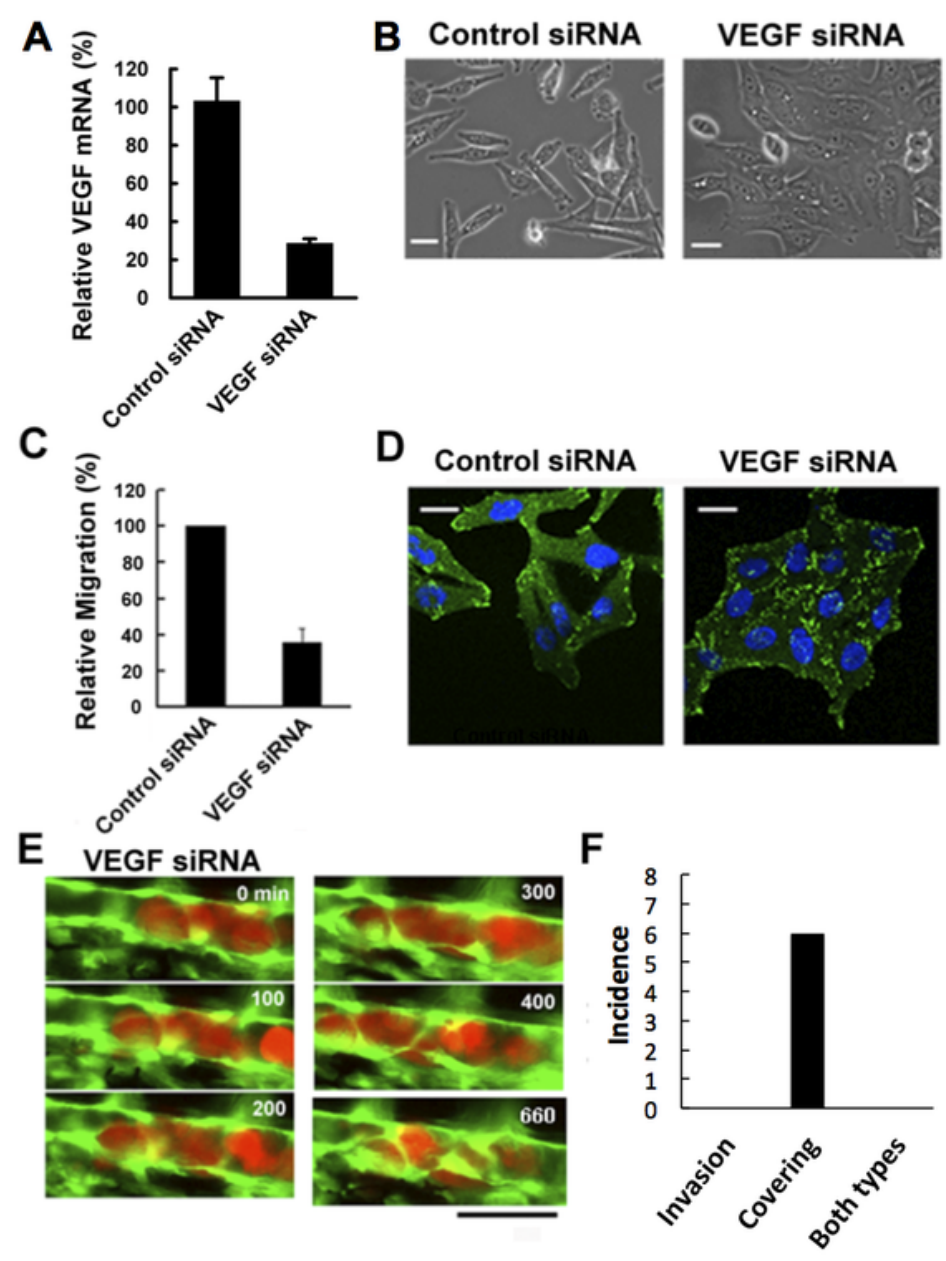




\section{Figure 4}

Effects of Anti-angiogenic Inhibitor, Sunitinib, on the Vasculature of Zebrafish Larvae and Cancer Cell Extravasation.

A, Intersegmental vessels were severely deteriorated by treatment with sunitinib. B, Extravasation of RFP-HeLa cells in the presence of sunitinib. The numbers indicate the elapsed time in minutes. C, The incidence of the 2 processes of extravasation. Seven larvae in which RFP-HeLa cells formed severe emboli were observed in the presence of sunitinib. Nine extravasation events were counted during $11 \mathrm{~h}$ observations. D, The sum of the area of extravasated cancer cell images was calculated from all of the 7 movies recorded in RFPHeLa cells with and without sunitinib treatments. Bars, $100 \mu \mathrm{m}$ (A) or $40 \mu \mathrm{m}$ (B). E and G, Scanning electron micrographs of the arteries in the control and sunitinib-treated larvae. Sunitinib-treated larvae showed thicker vascular walls. $\mathbf{F}$, The magnified image of the luminal face on the endothelial wall of a control larva. The endothelial wall showed many holes or fenestration-like structures (arrowhead). $\mathbf{H}$, The magnified image of the luminal face on the endothelial wall of a sunitinib-treated larva. The endothelial wall showed no fenestration-like structures. Bars, $10 \mu \mathrm{m}$ ( $\mathrm{E}$ and $\mathrm{G}$ ) or $0.5 \mu \mathrm{m}(\mathrm{F}$ and $\mathrm{H})$. 


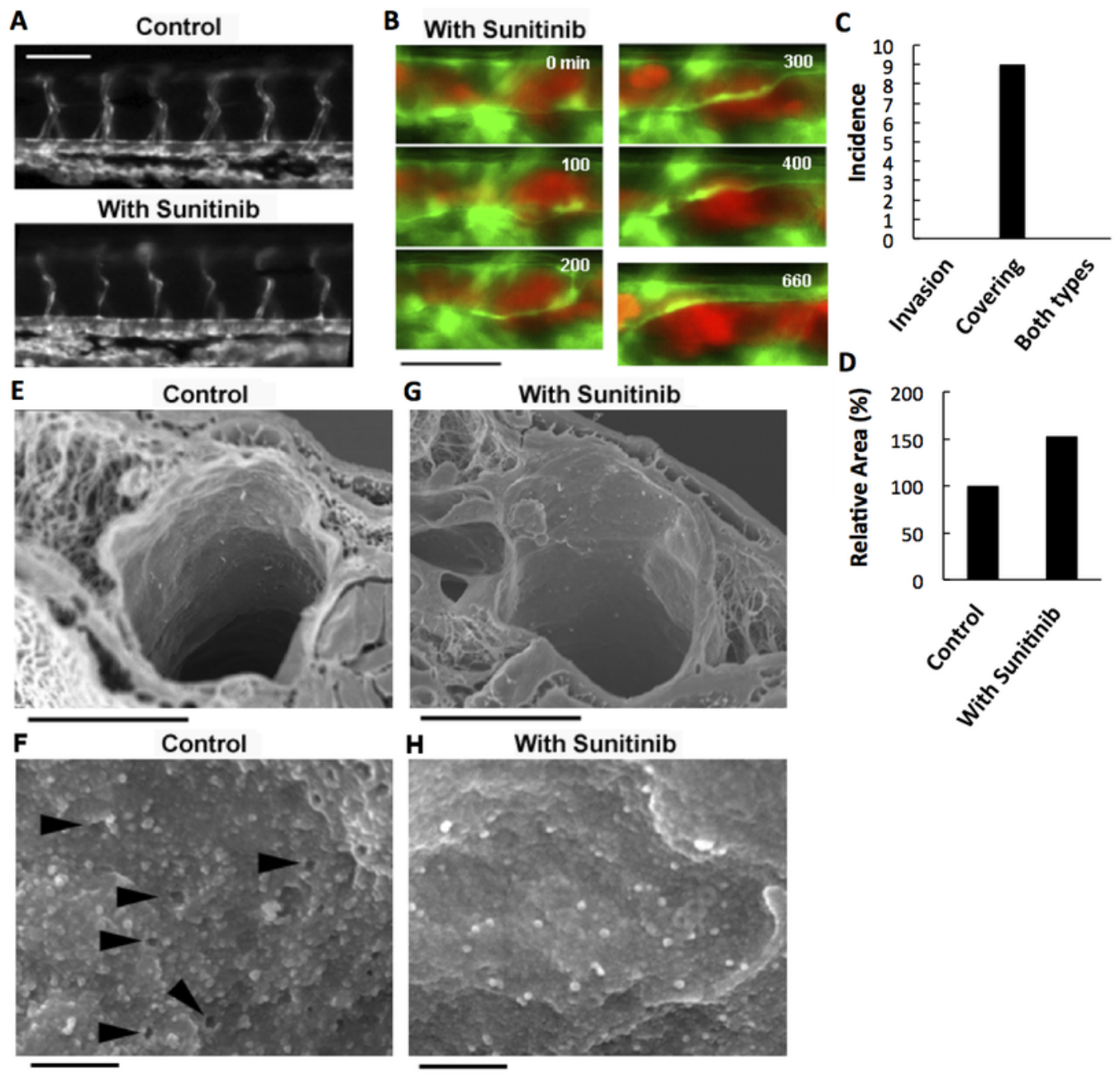




\section{Figure 5}

Model of the endothelial coverage regulation via vascular homeostasis maintenance.

Active EC in the normal vasculature, which is VEGF-dependent and fenestrated, and quiescent EC, which is VEGF-independent and unfenestrated, coexist in the same blood vessel. VEGF-targeted inhibitors only affect the active ECs, thus resulting in deterioration of new vasculature via apoptosis or vascular maturation. In contrast, the quiescent ECs that contain mature vasculature are reactivated independently of VEGF in order to maintain vascular homeostasis.

New Vasculature

Active EC
(VEGF Dependent,
Fenestrated)

Mature Vasculature

\section{Quiescent EC} (VEGF Independent, Unfenestrated)

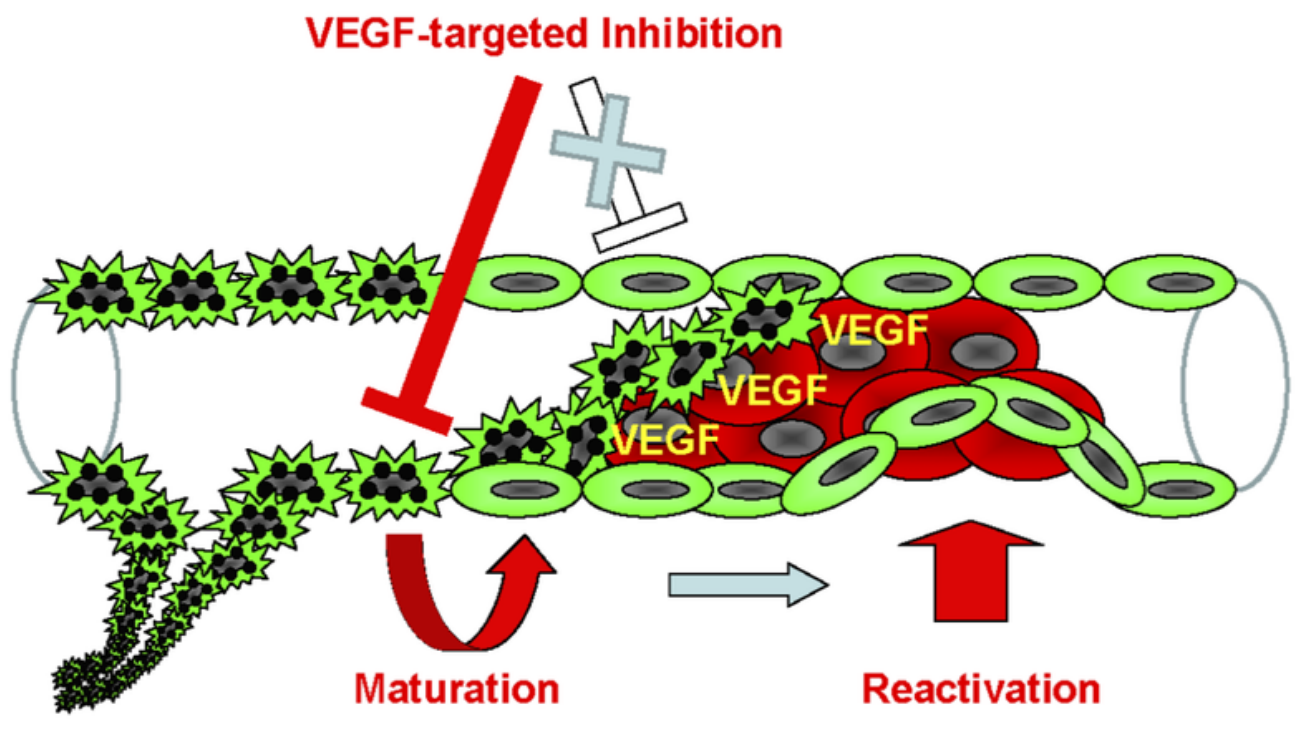

